TRANSACTIONS OF THE

AMERICAN MATHEMATICAL SOCIETY

Volume 352, Number 6, Pages 2479-2489

S 0002-9947(99)02331-4

Article electronically published on July 9, 1999

\title{
AVOIDABLE ALGEBRAIC SUBSETS OF EUCLIDEAN SPACE
}

\author{
JAMES H. SCHMERL
}

\begin{abstract}
Fix an integer $n \geq 1$ and consider real $n$-dimensional $\mathbb{R}^{n}$. A partition of $\mathbb{R}^{n}$ avoids the polynomial $p\left(x_{0}, x_{1}, \ldots, x_{k-1}\right) \in \mathbb{R}\left[x_{0}, x_{1}, \ldots, x_{k-1}\right]$, where each $x_{i}$ is an $n$-tuple of variables, if there is no set of the partition which contains distinct $a_{0}, a_{1}, \ldots, a_{k-1}$ such that $p\left(a_{0}, a_{1}, \ldots, a_{k-1}\right)=0$. The polynomial is avoidable if some countable partition avoids it. The avoidable polynomials are studied here. The polynomial $\|x-y\|^{2}-\|y-z\|^{2}$ is an especially interesting example of an avoidable one. We find (1) a countable partition which avoids every avoidable polynomial over $Q$, and (2) a characterization of the avoidable polynomials. An important feature is that both the "master" partition in (1) and the characterization in (2) depend on the cardinality of $\mathbb{R}$.
\end{abstract}

\section{INTRODUCTION}

A polynomial $p\left(x_{0}, x_{1}, \ldots, x_{k-1}\right) \in \mathbb{R}\left[x_{0}, x_{1}, \ldots, x_{k-1}\right]$, where $\mathbb{R}$ is the ordered field of reals and $n, k<\omega$, is a $(k, n)$-ary polynomial if each $x_{i}$ is an $n$-tuple of variables. A partition of $n$-dimensional Euclidean space $\mathbb{R}^{n}$ is said to avoid the $(k, n)$-ary polynomial $p\left(x_{0}, x_{1}, \ldots, x_{k-1}\right)$ if whenever $a_{0}, a_{1}, \ldots, a_{k-1} \in \mathbb{R}^{n}$ are distinct and in the same set of the partition, then $p\left(a_{0}, a_{1}, \ldots, a_{k-1}\right) \neq 0$. We say that the polynomial $p\left(x_{0}, x_{1}, \ldots, x_{k-1}\right)$ is avoidable if some countable partition avoids it. A polynomial that is not avoidable is unavoidable.

The question of which $(1, n)$-ary polynomials are avoidable is easily answered. If $p(x)$ is $(1, n)$-ary, then $p(x)$ is avoidable iff $p(x)$ has no zeros. Moreover, if $p(x)$ is avoidable, then every partition of $\mathbb{R}^{n}$ avoids it. Henceforth, even if it is not made explicit, the following proviso will be in force: all polynomials considered will be $(k, n)$-ary where $1 \leq n<\omega$ and $2 \leq k<\omega$. We will say that a polynomial is $(-, n)$-ary if it is $(k, n)$-ary, where $2 \leq k<\omega$.

Consider, as a nontrivial example, the $(3, n)$-ary polynomial $p(x, y, z)=$ $\|x-y\|^{2}-\|y-z\|^{2}$, where $\|w\|$ denotes the Euclidean norm of $w \in \mathbb{R}^{n}$. If $a, b, c \in \mathbb{R}^{n}$ are the vertices of an isosceles triangle with apex $b$, then $p(a, b, c)=0$. I proved a theorem in [16] that implies that $p(x, y, z)$ is avoidable, thereby answering a question of Erdôs about the avoidability of isosceles triangles. The method of proof of that theorem will be further exploited here, resulting in a complete characterization of avoidable polynomials. The following two theorems concerning polynomials over the field $\mathbb{Q}$ of rationals will be proved. The first of the theorems asserts the existence of what might be called, adapting terminology of [9], a master partition

Received by the editors November 5, 1997.

1991 Mathematics Subject Classification. Primary 03E15, 04A20.

Key words and phrases. Algebraic sets, avoidable polynomials, infinite combinatorics. 
of $\mathbb{R}^{n}$. The second one refers to the effectiveness of determining whether a given polynomial is avoidable.

Theorem 0.1. For each $n<\omega$ there is a countable partition of $\mathbb{R}^{n}$ which avoids every avoidable $(-, n)$-ary polynomial over $\mathbb{Q}$.

Theorem 0.2. The set of unavoidable polynomials over $\mathbb{Q}$ is recursively enumerable.

A subset $X \subseteq\left(\mathbb{R}^{n}\right)^{k}$ is algebraic if $X$ is the zero-set of some $(k, n)$-ary polynomial. Whether or not a polynomial is avoidable depends only on its zero-set, so the definition of avoidability can easily be extended to all $X \subseteq\left(\mathbb{R}^{n}\right)^{k}$. However, only algebraic sets will be considered in this paper.

There is a hitch in each of Theorems 0.1 and 0.2 . Even though Theorem 0.2 asserts that the set of unavoidable polynomials over $\mathbb{Q}$ is recursively enumerable, we cannot say exactly what that set is since it depends on the underlying set theory and, in particular, on the size of the continuum (that is, on $|\mathbb{R}|$, which is $\left.2^{\aleph_{0}}\right)$. Similarly, exactly which partition will do the job in Theorem 0.1 depends on the size of the continuum. In order to clarify the situation, the crucial concepts of $m$-avoidability, for $m<\omega$, and $\omega$-avoidability will be needed. In Definition 1.1]we define what it means for a $(k, n)$-ary polynomial to be $m$-avoidable. The notion of $m$-avoidability gets stricter as $m$ increases; thus, a polynomial which is $(m+1)$ avoidable is also $m$-avoidable (see Proposition 1.2). A polynomial is $\omega$-avoidable iff it is $m$-avoidable for each $m<\omega$; but also a $(k, n)$-ary polynomial is $\omega$-avoidable iff it is $(k-1)$-avoidable (see Proposition 1.3). The set of $m$-unavoidable polynomials over $\mathbb{Q}$ is recursively enumerable; in fact, it is r.e. uniformly in $m$. Moreover, the notion of $m$-avoidable is absolute: whether or not a given polynomial is $m$-avoidable is independent of the underlying set theory.

The following three theorems clarify and extend Theorems 0.1 and 0.2

Theorem 0.3 (Assume $2^{\aleph_{0}} \geq \aleph_{m}$ ). Every avoidable polynomial is m-avoidable.

Theorem 0.4 (Assume $2^{\aleph_{0}} \leq \aleph_{m}$ ). Every $m$-avoidable polynomial is avoidable. Furthermore, if $\mathbb{F} \subseteq \mathbb{R}$ is a countable subfield and $n<\omega$, then there is a countable partition of $\mathbb{R}^{n}$ which avoids every polynomial which is an $m$-avoidable $(-, n)$-ary polynomial over $\mathbb{F}$.

Theorem 0.5. Every $\omega$-avoidable polynomial is avoidable. Furthermore, if $\mathbb{F} \subseteq \mathbb{R}$ is a countable subfield and $n<\omega$, then there is a countable partition of $\mathbb{R}^{n}$ which avoids every polynomial which is an $\omega$-avoidable $(-, n)$-ary polynomial over $\mathbb{F}$.

Theorems 0.3 and 0.4 immediately imply the following corollary.

Corollary 0.6 (Assume $\left.2^{\aleph_{0}} \leq \aleph_{m}\right)$. A polynomial is avoidable iff it is m-avoidable.

Similarly, the following corollary is a consequence of Theorems 0.3 and 0.5

Corollary 0.7 (Assume $2^{\aleph_{0}} \leq \aleph_{\omega}$ ). A polynomial is avoidable iff it is $\omega$-avoidable.

It is evident that Theorem 0.1 is a consequence of Theorems 0.30 .5 In fact, Theorem 0.1 follows from Corollary 0.6 and the second part of Theorem 0.4 if $2^{\aleph_{0}}>$ $\aleph_{\omega}$, and it follows from Corollary 0.7 and the second part of Theorem 0.5 otherwise. Once the appropriate definitions are made, it will be evident that Theorems 0.30 .5 also imply Theorem 0.2 . 


\section{Definitions And EXAmples}

The crucial notions of $m$-avoidable and $\omega$-avoidable polynomials are defined in this section.

If $\mathbb{F} \subseteq \mathbb{R}$ and $X \subseteq \mathbb{R}^{m}$, then $X$ is $\mathbb{F}$-definable if $X$ is definable by a first-order formula in the language of ordered fields allowing parameters from $\mathbb{F}$. We say that $X$ is definable if $X$ is $\mathbb{R}$-definable. A subset $X \subseteq \mathbb{R}^{m}$ is semi-algebraic if it is a finite Boolean combination of sets of the form $\left\{x \in \mathbb{R}^{m}: p(x) \geq 0\right\}$, where $p(x)$ is an $m$ ary polynomial. By Tarski's theorem on the elimination of quantifiers, the definable sets are precisely the semi-algebraic ones. The survey article [17] is recommended as a source of information on Tarski's theorem and definable subsets of $\mathbb{R}^{m}$. The book [1] is recommended for a more thorough treatment of semi-algebraic sets.

We say that a function $\alpha: A_{0} \times A_{1} \times \cdots \times A_{m-1} \rightarrow B_{0} \times B_{1} \times \cdots \times B_{m-1}$ is coordinately induced if there are functions $\alpha_{0}: A_{0} \rightarrow B_{0}, \alpha_{1}: A_{1} \rightarrow B_{1}, \ldots, \alpha_{m-1}: A_{m-1}$ $\rightarrow B_{m-1}$ such that $\alpha\left(a_{0}, a_{1}, \ldots, a_{m-1}\right)=\left\langle\alpha_{0}\left(a_{0}\right), \alpha_{1}\left(a_{1}\right), \ldots, \alpha_{m-1}\left(a_{m-1}\right)\right\rangle$ whenever $\left\langle a_{0}, a_{1}, \ldots, a_{m-1}\right\rangle \in A_{0} \times A_{1} \times \cdots \times A_{m-1}$. A function $g: A^{m} \rightarrow B$ is oneone in each coordinate if whenever $a_{0}, a_{1}, \ldots, a_{m-1} \in A$ and $a_{i} \neq a_{i}^{\prime} \in A$, then $g\left(a_{0}, a_{1}, \ldots, a_{m-1}\right) \neq g\left(a_{0}, a_{1}, \ldots, a_{i-1}, a_{i}^{\prime}, a_{i+1}, \ldots, a_{m-1}\right)$.

Definition 1.1. Let $n<\omega$ and $2 \leq k<\omega$ and suppose $p\left(x_{0}, x_{1}, \ldots, x_{k-1}\right)$ is a $(k, n)$-ary polynomial.

(1) For each $m<\omega$, we say that $p\left(x_{0}, x_{1}, \ldots, x_{k-1}\right)$ is $m$-avoidable if for each definable function $g:(0,1)^{m} \rightarrow \mathbb{R}^{n}$ which is one-one in each coordinate and for distinct $e_{0}, e_{1}, \ldots, e_{k-1} \in(0,1)^{m}$, there is a coordinately induced $\alpha:(0,1)^{m} \rightarrow$ $(0,1)^{m}$ such that $p\left(g \alpha\left(e_{0}\right), g \alpha\left(e_{1}\right), \ldots, g \alpha\left(e_{k-1}\right)\right) \neq 0$.

(2) $p\left(x_{0}, x_{1}, \ldots, x_{k-1}\right)$ is $\omega$-avoidable if $p\left(x_{0}, x_{1}, \ldots, x_{k-1}\right)$ is $m$-avoidable for each $m<\omega$.

In Definition 1.1(1) it makes sense to consider $m=0$ : if $k \geq 2$, then each $(k, n)$-ary polynomial is vacuously 0 -avoidable. This case is of no real interest, so we will tacitly assume that $m>0$. Notice that there is some flexibility possible in Definition [1.1 1). For example, we could use any other open $m$-box as the domain of $g$, and we could take the $e_{i}$ 's to be from $\mathbb{R}^{m}$ (or even $X^{m}$ for any infinite set $X)$. Moreover, if it is further required of $g$ that it be analytic, then the notion of an $m$-avoidable polynomial remains unchanged. (See the proof of Theorem 0.4 and Section 2.4. of [1].)

It easily follows from Definition 1.1 and Tarski's theorem on the decidability of the theory $\operatorname{Th}(\mathbb{R},+, \cdot, \leq, 0,1)$ (see [17]) that the set of $m$-unavoidable polynomials over $\mathbb{Q}$ is a recursively enumerable set (uniformly in $m$ ). From this it is evident that Theorems 0.30 .5 imply Theorem 0.2 I conjecture that the set of $m$-avoidable polynomials over $\mathbb{Q}$ actually is recursive uniformly in $m$, thereby implying that a similar improvement to Theorem 0.2 would hold. This conjecture is discussed in §3.

The next two propositions state some nearly immediate consequences of Definition 1.1

Proposition 1.2. Suppose that $r<m<\omega$. Then every $m$-avoidable polynomial is r-avoidable.

Proof. Without loss of generality, we can suppose that $m=r+1$ and that $p\left(x_{0}, x_{1}, \ldots, x_{k-1}\right)$ is $(r+1)$-avoidable. We will show that it is $r$-avoidable. Let $g:(0,1)^{r} \rightarrow \mathbb{R}^{n}$ be a definable function which is one-one in each coordinate, and 
also let $e_{0}, e_{1}, \ldots, e_{k-1} \in(0,1)^{r}$ be distinct. Now let $h:(0,1)^{r+1} \rightarrow \mathbb{R}^{n}$ be defined so that $h\left(y_{0}, y_{1}, \ldots, y_{r}\right)=g\left(y_{0}, y_{1}, \ldots, y_{r-2}, \frac{1}{2}\left(y_{r-1}+y_{r}\right)\right)$. Clearly, $h$ is definable and one-one in each coordinate. For each $i<k$, let $e_{i}^{\prime}=e_{i}{ }^{\wedge}\left\langle\frac{1}{2}\right\rangle \in$ $(0,1)^{r+1}$, and note that $e_{0}^{\prime}, e_{1}^{\prime}, \ldots, e_{k-1}^{\prime}$ are distinct. Thus, there is a coordinately induced $\beta:(0,1)^{r+1} \rightarrow(0,1)^{r+1}$ such that $p\left(h \beta\left(e_{0}^{\prime}\right), h \beta\left(e_{1}^{\prime}\right), \ldots, h \beta\left(e_{k-1}^{\prime}\right)\right) \neq 0$. Let $\beta\left(y_{0}, y_{1}, \ldots, y_{r}\right)=\left\langle\beta_{0}\left(y_{0}\right), \beta_{1}\left(y_{1}\right), \ldots, \beta_{r}\left(y_{r}\right)\right\rangle$, and then let $\alpha:(0,1)^{r} \rightarrow(0,1)^{r}$ be such that

$$
\alpha\left(y_{0}, y_{1}, \ldots, y_{r-1}\right)=\left\langle\beta_{0}\left(y_{0}\right), \beta_{1}\left(y_{1}\right), \ldots, \beta_{r-2}\left(y_{r-2}\right), \frac{1}{2}\left(\beta_{r-1}\left(y_{r-1}\right)+\beta_{r}\left(\frac{1}{2}\right)\right)\right\rangle .
$$

Clearly, $\alpha$ is coordinately induced, and $g \alpha\left(e_{i}\right)=h \beta\left(e_{i}^{\prime}\right)$ for each $i<k$. Thus, $p\left(g \alpha\left(e_{0}\right), g \alpha\left(e_{1}\right), \ldots, g \alpha\left(e_{k-1}\right)\right) \neq 0$.

Proposition 1.3. Suppose that $2 \leq k<\omega$ and that $p\left(x_{0}, x_{1}, \ldots, x_{k-1}\right)$ is a $(k, n)$ ary polynomial. Then $p\left(x_{0}, x_{1}, \ldots, x_{k-1}\right)$ is $\omega$-avoidable iff $p\left(x_{0}, x_{1}, \ldots, x_{k-1}\right)$ is $(k-1)$-avoidable.

Proof. Suppose that $p\left(x_{0}, x_{1}, \ldots, x_{k-1}\right)$ is $(k-1)$-avoidable and that $2 \leq k \leq$ $m<\omega$. We will show that it is $m$-avoidable. Let $g:(0,1)^{m} \rightarrow \mathbb{R}^{n}$ be a definable function which is one-one in each coordinate. Let $e_{0}, e_{1}, \ldots, e_{k-1} \in(0,1)^{m}$ be distinct. It is easy to see that there is a set of $k-1$ coordinates on which the $e_{i}$ are distinct. Without loss of generality, assume that these are the first $k-1$ coordinates, and let $\pi:(0,1)^{m} \rightarrow(0,1)^{k-1}$ be the projection onto the first $k-1$ coordinates. Let $\beta:(0,1)^{k-1} \rightarrow(0,1)^{m}$ be such that $\beta\left(y_{0}, y_{1}, \ldots, y_{k-2}\right)=$ $\left\langle y_{0}, y_{1}, \ldots, y_{k-2}, \frac{1}{2}, \frac{1}{2}, \ldots, \frac{1}{2}\right\rangle$. Since $g \beta:(0,1)^{k-1} \rightarrow \mathbb{R}^{n}$ is definable and one-one in each coordinate, there is a coordinately induced $\alpha:(0,1)^{k-1} \rightarrow(0,1)^{k-1}$ such that $p\left(g \beta \alpha \pi\left(e_{0}\right), g \beta \alpha \pi\left(e_{1}\right), \ldots, g \beta \alpha \pi\left(e_{k-1}\right)\right) \neq 0$. But $\beta \alpha \pi:(0,1)^{m} \rightarrow(0,1)^{m}$ is coordinately induced.

We say that a $(k, n)$-ary polynomial $p\left(x_{0}, x_{1}, \ldots, x_{k-1}\right)$ is irreflexive if

$$
p(a, a, \ldots, a) \neq 0
$$

for each $a \in \mathbb{R}^{n}$. I proved in [15] that every irreflexive $(k, n)$-ary polynomial is avoidable. Earlier, Komjáth [9] had obtained this result as a consequence of the Continuum Hypothesis $(\mathrm{CH})$. It is evident that every irreflexive $(k, n)$-ary polynomial is $\omega$-avoidable. For, in Definition [1.1 (1), no matter what $g$ and $e_{0}, e_{1}, \ldots, e_{k-1}$ are, just pick any $a \in(0,1)$ and then let $\alpha:(0,1)^{m} \rightarrow(0,1)^{m}$ be the constant function $(a, a, \ldots, a)$. Hence, Theorem 0.5 easily implies that every irreflexive polynomial is avoidable.

Perhaps the earliest result concerning avoidable polynomials is Cantor's theorem that $2^{\aleph_{0}}>\aleph_{0}$. It is evident that Cantor's theorem can be restated as a property of the $(2,1)$-ary polynomial which is identically 0 : this polynomial is unavoidable iff $2^{\aleph_{0}}>\aleph_{0}$. Notice that this polynomial is not 1 -avoidable.

It is easy to see that for every $k \geq 2$, each nonzero $(k, 1)$-ary polynomial is 1 avoidable. In particular, if $r_{0}, r_{1}, \ldots, r_{k-1} \in \mathbb{Q}$ are not all 0 , then the $(k, 1)$-ary polynomial $r_{0} x_{0}+r_{1} x_{1}+\cdots+r_{k-1} x_{k-1}$ is 1 -avoidable. It had been proved by Erdős and Kakutani [6], assuming $\mathrm{CH}$, that $\mathbb{R}$ can be partitioned into countably many sets each one of which is linearly independent over $\mathbb{Q}$. In other words, if $\mathrm{CH}$, then there is a single countable partition of $\mathbb{R}$ which avoids each of the nonzero polynomials $r_{0} x_{0}+r_{1} x_{1}+\cdots+r_{k-1} x_{k-1}$ over $\mathbb{Q}$. This result is an easy consequence of Theorem 0.4 
Komjáth [10 proved that for each $n$ there is a countable partition of $\mathbb{R}^{n}$ such that no two distinct points in the same set of the partition are at a rational distance. Earlier, Erdős and Komjáth [7] had obtained this result for $n=3$, and earlier still Erdős and Hajnal [4] had obtained it for $n=2$. (When $n=1$, the result is trivial.) For each $d$, where $0<d \in \mathbb{Q}$, consider the $(2, n)$-ary polynomial $p(x, y)=$ $\|x-y\|^{2}-d^{2}$. Notice that $p(a, b)=0$ just in the case the Euclidean distance between $a$ and $b$ is $d$. The Erdös-Komjáth result can be restated as follows: for each $n$ there is a countable partition of $\mathbb{R}^{n}$ which avoids each $(2, n)$-ary polynomial $\|x-y\|^{2}=d^{2}$ where $0<d \in \mathbb{Q}$. Of course, each such polynomial is irreflexive and consequently $\omega$-avoidable.

It is easy to see that the $(3, n)$-ary polynomial $p(x, y, z)=\|x-y\|^{2}-\|y-z\|^{2}$ is $\omega$-avoidable. By Proposition 1.3. we need only show that it is 2-avoidable. Consider a definable function $g:(0,1)^{2} \rightarrow \mathbb{R}^{n}$ which is one-one in each coordinate, and also consider distinct $e_{0}, e_{1}, e_{2} \in(0,1)^{2}$. Since $e_{0} \neq e_{2}$, we can assume without loss of generality that $e_{00} \neq e_{20}$, and then we can also assume, without loss of generality, that $e_{00} \neq e_{10}$. Then there is a coordinately induced $\alpha:(0,1)^{2} \rightarrow(0,1)^{2}$ such that $\alpha\left(e_{0}\right)=e_{0}$ and $\alpha\left(e_{1}\right)=\alpha\left(e_{2}\right)=e_{1}$. Since $g$ is one-one in each coordinate, there is a coordinately induced $\beta:(0,1)^{2} \rightarrow(0,1)^{2}$ such that $\beta\left(e_{0}\right) \neq e_{1}=\beta\left(e_{1}\right)$ and $g\left(\beta\left(e_{0}\right)\right) \neq g\left(\beta\left(e_{1}\right)\right)$. Then $p\left(g \beta \alpha\left(e_{0}\right), g \beta \alpha\left(e_{1}\right), g \beta \alpha\left(e_{2}\right)\right) \neq 0$, proving that $p(x, y, z)$ is $\omega$-avoidable.

As mentioned previously, I had proved in [16] that the $(3, n)$-ary polynomial $\|x-y\|^{2}-\|y-z\|^{2}$ is avoidable, thereby showing that isosceles triangles are avoidable. This result, which is now seen to follow from Theorem [0.5, improved on various partial results in [2], [3], 8], [13, [14], [15].

Whether or not a polynomial is avoidable depends only on its zero-set. Similarly, whether or not a polynomial is $m$-avoidable depends only on its zero-set. Consider a set $\mathcal{P}$ of $(k, n)$-ary polynomials. According to the Hilbert Basis Theorem, there is a finite subset $\mathcal{P}_{0} \subseteq \mathcal{P}$ whose zero-set is the zero-set of $\mathcal{P}$, and then there is a single polynomial $p\left(x_{0}, x_{1}, \ldots, x_{k-1}\right)$, which can be taken to be the sum of the squares of polynomials in $\mathcal{P}$, whose zero-set is the same as the zero-set of $\mathcal{P}$. We will say that $\mathcal{P}$ is $m$-avoidable just in case $p\left(x_{0}, x_{1}, \ldots, x_{k-1}\right)$ is $m$-avoidable.

In the next two propositions we give examples of (sets of) polynomials which are $m$-avoidable but not $(m+1)$-avoidable.

Proposition 1.4. Suppose $2 \leq n<\omega$. For $0<i<j \leq n$, consider the $(n+1, n)$ ary polynomial $p_{i j}\left(x_{0}, x_{1}, \ldots, x_{n}\right)=\left\|x_{i}-x_{0}\right\|^{2}+\left\|x_{j}-x_{0}\right\|^{2}-\left\|x_{i}-x_{j}\right\|^{2}$, and let $\mathcal{P}=\left\{p_{i j}\left(x_{0}, x_{1}, \ldots, x_{n}\right): 0<i<j \leq n\right\}$. Then $\mathcal{P}$ is $(n-1)$-avoidable but not $n$-avoidable.

Proof. First, we show that $\mathcal{P}$ is not $n$-avoidable. Let $g:(0,1)^{n} \rightarrow(0,1)^{n}$ be the identity function. Let $0<a<b<1$, and then let $e_{0}=(0,0, \ldots, 0)$ and for $1 \leq i \leq n$, let $e_{i}=(0,0, \ldots, 0,1,0,0, \ldots, 0)$ (which consists of $i-10$ 's followed by 1 and then followed by all 0 's). Notice that if $0<i<j \leq n$, then $e_{0}, e_{i}, e_{j}$ are the vertices of a right triangle with right angle at $e_{0}$. If $\alpha: \mathbb{R}^{n} \rightarrow \mathbb{R}^{n}$ is coordinately induced, then $\alpha\left(e_{0}\right), \alpha\left(e_{i}\right), \alpha\left(e_{j}\right)$ still are the vertices of a (possibly degenerate) right triangle with right angle at $\alpha\left(e_{0}\right)$, so $p_{i j}\left(\alpha\left(e_{0}\right), \alpha\left(e_{i}\right), \alpha\left(e_{j}\right)\right)=0$. This shows that $\mathcal{P}$ is not $n$-avoidable.

To prove that $\mathcal{P}$ is $(n-1)$-avoidable, let $g:(0,1)^{n-1} \rightarrow \mathbb{R}^{n}$ be a definable function which is one-one in each coordinate. Let $e_{0}, e_{1}, \ldots, e_{n} \in(0,1)^{n-1}$ be distinct. Whenever $1 \leq i \leq n$, there is $k<n-1$ such that $e_{i k} \neq e_{0 k}$. Therefore, 
there are $i, j, k$ such that $1 \leq i<j \leq n, k<n-1$ and $e_{i k} \neq e_{0 k} \neq e_{j k}$. Then there is a coordinately induced $\alpha:(0,1)^{n-1} \rightarrow(0,1)^{n-1}$ such that $\alpha\left(e_{i}\right)=\alpha\left(e_{j}\right)$ and $\left(\alpha\left(e_{j}\right)\right)_{r}=\left(\alpha\left(e_{0}\right)\right)_{r}$ iff $r \neq k$. Hence, $g \alpha\left(e_{i}\right)=g \alpha\left(e_{j}\right) \neq g \alpha\left(e_{0}\right)$, so clearly $p_{i j}\left(g \alpha\left(e_{0}\right), g \alpha\left(e_{i}\right), g \alpha\left(e_{j}\right)\right) \neq 0$.

The zero-set of $\mathcal{P}$ in Proposition 1.4 has a clear geometric interpretation. For a given $n \geq 2$, if $\left\langle a_{0}, a_{1}, \ldots, a_{n}\right\rangle \in \mathbb{R}^{n+1}$, then $\left\langle a_{0}, a_{1}, \ldots, a_{n}\right\rangle$ is in the zero-set of $\mathcal{P}$ iff $a_{0}, a_{1}, \ldots, a_{n}$ are the vertices of a (possibly degenerate) right simplex with all angles at $a_{0}$ being right angles. Erdös and Komjáth [7] proved that $\mathrm{CH}$ is equivalent to the avoidability of the $(3,2)$-ary polynomial $\|x-y\|^{2}+\|z-y\|^{2}-\|x-z\|^{2}$.

The polynomials in the proof of the next proposition were used by Komjáth [11] for a purpose similar to the one we are using them for.

Proposition 1.5. For each $n<\omega$ there is an $(n+1)$-avoidable, but not $(n+2)$ avoidable, set of $\left(2^{n+2}, 1\right)$-ary linear polynomials.

Proof. Let $n<\omega$, and for each $f: n+2 \rightarrow 2$ let $x_{f}$ be a variable. For functions $f, h, p, q: n+2 \rightarrow 2$ and $r<n+2$, where

$$
f(i)=h(i) \quad \text { and } \quad p(i)=q(i), \text { if } i \neq r,
$$

and

$$
f(r)=p(r)=0 \quad \text { and } \quad h(r)=q(r)=1,
$$

consider the $\left(2^{n+2}, 1\right)$-ary polynomial $x_{f}-x_{h}-x_{p}+x_{q}$. Let $\mathcal{P}$ be the set of all such polynomials. We will show that $\mathcal{P}$ is $(n+1)$-avoidable but not $(n+2)$-avoidable.

To see that $\mathcal{P}$ is not $(n+2)$-avoidable, let $g: \mathbb{R}^{n+2} \rightarrow \mathbb{R}$ be defined by

$$
g\left(y_{0}, y_{1}, \ldots, y_{n+1}\right)=y_{0}+y_{1}+\cdots+y_{n+1} .
$$

Clearly, $g$ is definable and one-one in each coordinate. For $f: n+2 \rightarrow 2$, let $e_{f}=\langle f(0), f(1), \ldots, f(n+1)\rangle$, and let $\alpha:(0,1)^{n+2} \rightarrow(0,1)^{n+2}$ be coordinately induced. Consider a polynomial $x_{f}-x_{h}-x_{p}+x_{q}$ in $\mathcal{P}$, where $f(r)=p(r)=0$ and $h(r)=q(r)=1$. Since, for $i \neq r,\left(e_{f}\right)_{i}=f(i)=h(i)=\left(e_{h}\right)_{i}$ and $\left(e_{p}\right)_{i}=$ $p(i)=q(i)=\left(e_{q}\right)_{i}$, it follows that $\left(\alpha\left(e_{f}\right)\right)_{i}=\alpha_{i}\left(\left(e_{f}\right)_{i}\right)=\alpha_{i}\left(\left(e_{h}\right)_{i}\right)=\left(\alpha\left(e_{h}\right)\right)_{i}$ and $\left(\alpha\left(e_{p}\right)\right)_{i}=\alpha_{i}\left(\left(e_{p}\right)_{i}\right)=\alpha_{i}\left(\left(e_{q}\right)_{i}\right)=\left(\alpha\left(e_{q}\right)\right)_{i}$. Therefore,

$$
\begin{aligned}
& g \alpha\left(e_{f}\right)-g \alpha\left(e_{h}\right)-g \alpha\left(e_{p}\right)+g \alpha\left(e_{q}\right) \\
& \quad=\left(\alpha\left(e_{f}\right)\right)_{r}-\left(\alpha\left(e_{h}\right)\right)_{r}-\left(\alpha\left(e_{p}\right)\right)_{r}+\left(\alpha\left(e_{q}\right)\right)_{r} \\
& =\alpha_{r}(0)-\alpha_{r}(1)-\alpha_{r}(0)-\alpha_{r}(1)=0 .
\end{aligned}
$$

This proves $\mathcal{P}$ is not $(n+2)$-avoidable.

To see that $\mathcal{P}$ is $(n+1)$-avoidable, let $g:(0,1)^{n+1} \rightarrow \mathbb{R}$ be a definable function which is one-one in each coordinate, and let $e_{f} \in(0,1)^{n+1}$, for $f: n+2 \rightarrow 2$, be distinct. There is $j<n+1$ such that $\left|\left\{\left(e_{f}\right)_{j}: f: n+2 \rightarrow 2\right\}\right| \geq 3$, and then for that $j$ there is some $a \in(0,1)$ such that $1 \leq\left|\left\{f:\left(e_{f}\right)_{j}=a, f: n+2 \rightarrow 2\right\}\right|<$ $2^{n+1}$. Then, there are $r<n+2$ and $f, h$ such that $f(i)=h(i)$, when $i \neq r$, and $f(r)=0$ and $h(r)=1$, and for just one of these functions (say $f$ ) it is the case that $\left(e_{f}\right)_{r}=a$. Then there are also $p, q$ such that $\left(e_{p}\right)_{r} \neq a \neq\left(e_{q}\right)_{r}$ and $p(i)=q(i)$, when $i \neq r$, and $p(r)=0$ and $q(r)=1$. Clearly, there is a coordinately induced $\alpha:(0,1)^{n+1} \rightarrow(0,1)^{n+1}$ such that $\alpha\left(e_{f}\right) \neq \alpha\left(e_{h}\right)=\alpha\left(e_{p}\right)=\alpha\left(e_{q}\right)$, and $\alpha_{i}\left(e_{f}\right)=\alpha_{i}\left(e_{h}\right)$ for $i \neq r$. Then $g \alpha\left(e_{f}\right)-g \alpha\left(e_{h}\right)-g \alpha\left(e_{p}\right)+g \alpha\left(e_{q}\right) \neq 0$ since $q \alpha\left(e_{f}\right) \neq g \alpha\left(e_{h}\right)=g \alpha\left(e_{p}\right)=g \alpha\left(e_{q}\right)$. This proves $\mathcal{P}$ is $(n+1)$-avoidable. 
In both Propositions 1.4 and 1.5 when proving $\mathcal{P}$ was $m$-avoidable, we made no use of the fact that $g$ was definable. I conjecture that the omission of this condition on $g$ in Definition 1.1 would not have any affect on the notion of $m$-avoidability. See $\$ 3$ for more on this conjecture.

\section{Characterizing AVOidability}

In this section Theorems $0.3,0.4$ and 0.5 will be proved. As previously noted, Theorems 0.1 and 0.2 are consequences of these theorems.

For the proof of Theorem 0.3 , we need a special case of the polarized partition theorem of Erdős and Hajnal. Although this theorem is not explicitly stated in the comprehensive book [5, similar results can be found there. A proof can be found in [11. However, for the sake of completeness, the short proof of this result will be given here. We state it first, in the conventional way, as Lemma 2.1, and then restate it as Corollary 2.2 in an equivalent way but one which is better suited for our purposes.

Lemma 2.1. Suppose $k, m<\omega,|A| \geq \aleph_{m}$, and $F: A^{m} \rightarrow \omega$. Then there are $B_{0}, B_{1}, \ldots, B_{m-1} \subseteq A$ such that $\left|B_{0}\right|=\left|B_{1}\right|=\cdots=\left|B_{m-1}\right|=k$ and $F$ is constant on $B_{0} \times B_{1} \times \cdots \times B_{m-1}$.

Proof. The proof is by induction on $m$. When $m=0$, the lemma is vacuously true, and when $m=1$, it is trivial. Assume that $m \geq 1$ and that the lemma is true for $m$. Let $F=A^{m+1} \rightarrow \omega$, where $|A| \geq \aleph_{m+1}$. Let $A_{0} \subseteq A$ be such that $\left|A_{0}\right|=\aleph_{m}$. By the inductive hypothesis, for each $a \in A$ there is a cube $B_{0} \times B_{1} \times \cdots \times B_{m-1} \subseteq A_{0}^{m}$, where $\left|B_{0}\right|=\left|B_{1}\right|=\cdots=\left|B_{m-1}\right|=k$, and there is $c<\omega$ such that $F$ has the constant value $c$ on $B_{0} \times B_{1} \times \cdots \times B_{m-1} \times\{a\}$. Since $|A|>\aleph_{m}$, there is $B_{m} \subseteq A_{0}$ such that $\left|B_{m}\right|=k$ and there are $c<\omega$ and a cube $B_{0} \times B_{1} \times \cdots \times B_{m-1} \subseteq A_{0}^{m}$ such that $F$ has the constant value $c$ on $B_{0} \times B_{1} \times \cdots \times B_{m-1} \times\{a\}$ for each $a \in B_{m}$. Then $F$ is constant on $B_{0} \times B_{1} \times \cdots \times B_{m}$.

Corollary 2.2. Suppose $k, m<\omega$, and $\left|A_{0}\right|,\left|A_{1}\right|, \ldots,\left|A_{m-1}\right| \geq \aleph_{m}$. Let $F: A_{0} \times$ $A_{1} \times \cdots \times A_{m-1} \rightarrow \omega$ and $e_{0}, e_{1}, \ldots, e_{k-1} \in A_{0} \times A_{1} \times \cdots \times A_{m-1}$. Then there is a coordinately induced injection $\alpha: A_{0} \times A_{1} \times \cdots \times A_{m-1} \rightarrow A_{0} \times A_{1} \times \cdots \times A_{m-1}$ such that $F \alpha\left(e_{0}\right)=F \alpha\left(e_{1}\right)=\cdots=F \alpha\left(e_{k-1}\right)$.

Proof of Theorem 0.3 . Let $p\left(x_{0}, x_{1}, \ldots, x_{k-1}\right) \in \mathbb{R}\left[x_{0}, x_{1}, \ldots, x_{k-1}\right]$ be an avoidable $(k, n)$-ary polynomial, and let $\chi: \mathbb{R}^{n} \rightarrow \omega$ be a countable partition which avoids it. With the intent of proving $p\left(x_{0}, x_{1}, \ldots, x_{k-1}\right)$ is $m$-avoidable, consider a definable function $g:(0,1)^{m} \rightarrow \mathbb{R}^{n}$ which is one-one in each coordinate. Also, consider distinct $e_{0}, e_{1}, \ldots, e_{k-1} \in(0,1)^{m}$. Then $\chi g:(0,1)^{m} \rightarrow \omega$ is a partition.

Let $\mathbb{F} \subseteq \mathbb{R}$ be a countable subfield such that $g$ is $\mathbb{F}$-definable. Let $B \subseteq(0,1)$ be a transcendence basis for $\mathbb{R}$ over $\mathbb{F}$, and let $B=B_{0} \cup B_{1} \cup \cdots \cup B_{m-1}$ be a partition of $B$ such that $\left|B_{0}\right|=\left|B_{1}\right|=\cdots=\left|B_{m-1}\right|=2^{\aleph_{0}} \geq \aleph_{m}$. By Corollary 2.2, there is a coordinately induced injection $\alpha:(0,1)^{m} \rightarrow B_{0} \times B_{1} \times \cdots \times B_{m-1}$ such that $\chi g \alpha\left(e_{0}\right)=\chi g \alpha\left(e_{1}\right)=\cdots=\chi g \alpha\left(e_{k-1}\right)$.

We next prove that $g \alpha:(0,1)^{m} \rightarrow \mathbb{R}^{n}$ is one-one. Let $\alpha_{0}, \alpha_{1}, \ldots, \alpha_{m-1}:(0,1) \rightarrow$ $B$ be such that $\alpha\left(x_{0}, x_{1}, \ldots, x_{m-1}\right)=\left\langle\alpha_{0}\left(x_{0}\right), \alpha_{1}\left(x_{1}\right), \ldots, \alpha_{m-1}\left(x_{m-1}\right)\right\rangle$ for each $\left\langle x_{0}, x_{1}, \ldots, x_{m-1}\right\rangle \in(0,1)^{m}$. Now suppose that

$$
g \alpha\left(x_{0}, x_{1}, \ldots, x_{m-1}\right)=g \alpha\left(y_{0}, y_{1}, \ldots, y_{m-1}\right),
$$


and, say, $x_{0} \neq y_{0}$. Clearly, each $\alpha_{i}$ is one-one, so $\alpha_{0}\left(x_{0}\right) \neq \alpha_{0}\left(y_{0}\right)$. Then

$$
g\left(\alpha_{0}\left(x_{0}\right), \alpha_{1}\left(x_{1}\right), \ldots, \alpha_{m-1}\left(x_{m-1}\right)\right)=g\left(\alpha_{0}\left(y_{0}\right), \alpha_{1}\left(y_{1}\right), \ldots, \alpha_{m-1}\left(y_{m-1}\right)\right) .
$$

Since $g$ is one-one in each coordinate, $\alpha_{0}\left(x_{0}\right)$ is the unique $b \in(0,1)$ such that $g\left(b, \alpha_{1}\left(x_{1}\right), \ldots, \alpha_{m-1}\left(x_{m-1}\right)\right)=g\left(\alpha_{0}\left(y_{0}\right), \alpha_{1}\left(y_{1}\right), \ldots, \alpha_{m-1}\left(y_{m-1}\right)\right)$, and therefore $\alpha_{0}\left(x_{0}\right)$ is $\left(\mathbb{F} \cup\left\{\alpha_{1}\left(x_{1}\right), \alpha_{2}\left(x_{2}\right), \ldots, \alpha_{m-1}\left(x_{m-1}\right), \alpha_{0}\left(y_{0}\right), \alpha_{1}\left(y_{1}\right), \ldots, \alpha_{m-1}\left(y_{m-1}\right)\right\}\right)$ definable. But $\alpha_{0}\left(x_{0}\right) \notin\left\{\alpha_{1}\left(x_{1}\right), \alpha_{2}\left(x_{2}\right), \ldots, \alpha_{m-1}\left(x_{m-1}\right), \alpha_{0}\left(y_{0}\right), \alpha_{1}\left(y_{1}\right), \ldots\right.$, $\left.\alpha_{m-1}\left(y_{m-1}\right)\right\}$, which is a contradiction. Thus, $g \alpha$ is one-one.

It follows that the coordinates $g \alpha\left(e_{0}\right), g \alpha\left(e_{1}\right), \ldots, g \alpha\left(e_{k-1}\right)$ are distinct. Since $\chi$ avoids $p\left(x_{0}, x_{1}, \ldots, x_{k-1}\right)$, it must be that $p\left(g \alpha\left(e_{0}\right), g \alpha\left(e_{1}\right), \ldots, g \alpha\left(e_{k-1}\right)\right) \neq 0$. Thus, $p\left(x_{0}, x_{1}, \ldots, x_{k-1}\right)$ is $m$-avoidable.

For the proof of Theorem 0.4 we will need a simple combinatorial lemma whose use was suggested by Komjáth [12 for a similar purpose. The set of finite subsets of $X$ is denoted by $[X]^{<\omega}$. If $(X,<)$ is a linearly ordered set and $a \in[X]^{<\omega}$, then $a_{i}$ is the $i$ th element of $a$ in the given order.

Lemma 2.3. Suppose $m<\omega$ and $(X,<)$ is a linearly ordered set such that $|X| \leq$ $\aleph_{m}$. Then there are functions $G:[X]^{<\omega} \rightarrow \omega$ and $H:[X]^{<\omega} \rightarrow[X]^{<\omega}$ such that, whenever $a, b \in[X]^{<\omega}$, the following hold:

(1) $H(a) \subseteq a$ and $|H(a)|=\min (|a|, m)$;

(2) if $G(a)=G(b)$ and $H(a)=H(b)$, then $a=b$;

(3) if $G(a)=G(b)$, then $|a|=|b|$ and for each $i<|H(a)|, a_{i} \in H(a)$ iff $b_{i} \in H(b)$.

Proof. We first show how to get $G$ and $H$ satisfying just (1) and (2). Without loss of generality we assume that $X=\omega_{m}$. (Keep in mind that for now only (1) and (2) are being considered, so the ordering on $X$ plays no role.) We will denote by $g_{m}$ and $h_{m}$ the functions $G$ and $H$ for this $m$. For $m=0$, just let $g_{0}$ be any injection and, of course, $h_{0}$ is the function which is constantly $\varnothing$. Now suppose we have $g_{m}$ and $h_{m}$, and we want $g_{m+1}$ and $h_{m+1}$. For each $\alpha<\omega_{m+1}$, let $F_{\alpha}: \alpha \rightarrow \omega_{m}$ be a one-one function. Consider $a \in\left[\omega_{m}\right]^{<\omega}$. If $a=\varnothing$, let $g_{m+1}(a)=0$ and $h_{m+1}=\varnothing$. Supposing $a \neq \varnothing$, let $\alpha=\max a$. Then let

$$
h_{m+1}(a)=\{\alpha\} \cup F_{\alpha}^{-1}\left(h_{m}\left(F_{\alpha}[a \backslash\{\alpha\}]\right)\right),
$$

and

$$
g_{m+1}(a)=g_{m}\left(F_{\alpha}[a \backslash\{\alpha\}]\right) .
$$

It is easy to see that these functions work.

Having $G$ and $H$ satisfying (1) and (2), we will modify $G$ so that in addition (3) is satisfied. We now are considering $(X,<)$ as a linearly ordered set. Let $\pi: \omega \times[\omega]^{<\omega} \times[\omega]^{<\omega} \rightarrow \omega$ be an injection. Let $F:[X]^{<\omega} \rightarrow[\omega]^{<\omega}$ be such that for $a \in[X]^{<\omega}, i \in F(a)$ iff $a_{i} \in H(a)$. Now replace $G$ with the function $a \mapsto$ $\langle G(a),|H(a)|, F(a)\rangle$. It is easy to see that $H$ and this new $G$ satisfy (1)-(3).

We will need another lemma which is a very slight modification of Lemma 7 in [16]. Since the proof is essentially unchanged from [16], we omit it here.

Lemma 2.4. Let $\mathbb{F} \subseteq \mathbb{R}$ be a subfield and $T$ a transcendence basis for $\mathbb{R}$ over $\mathbb{F}$. Let $D=\left(q_{0}, r_{0}\right) \times\left(q_{1}, r_{1}\right) \times \cdots \times\left(q_{k-1}, r_{k-1}\right) \subseteq \mathbb{R}^{k}$ be an $\mathbb{F}$-definable open $k$-box, and let $h: D \rightarrow \mathbb{R}$ be an $\mathbb{F}$-definable analytic function. Suppose that $\left(t_{0}, t_{1}, \ldots, t_{k-1}\right) \in T^{k} \cap$ $D$ is such that $h\left(t_{0}, t_{1}, \ldots, t_{k-1}\right)=0$. Further, suppose $\beta:\left\{t_{0}, t_{1}, \ldots, t_{k-1}\right\} \rightarrow \mathbb{R} i$ s such that $q_{i}<\beta\left(t_{i}\right)<r_{i}$ for each $i<k$. Then $h\left(\beta\left(t_{0}\right), \beta\left(t_{1}\right), \ldots, \beta\left(t_{k-1}\right)\right)=0$. 
Proof of Theorem 0.4 . Let $G:[\mathbb{R}]^{<\omega} \rightarrow \omega$ and $H:[\mathbb{R}]^{<\omega} \rightarrow[\mathbb{R}]^{<\omega}$ be as in Lemma [2.3, where we let $X=\mathbb{R}$ with the usual ordering on $\mathbb{R}$.

We can assume that $\mathbb{F}$ is a real-closed subfield of $\mathbb{R}$ (which is equivalent to its being relatively algebraically closed in $\mathbb{R}$ ). Let $T$ be a transcendence basis for $\mathbb{R}$ over $\mathbb{F}$. Following [16, for each $a=\left\langle a_{0}, a_{1}, \ldots, a_{n-1}\right\rangle \in \mathbb{R}^{n}$, we define the support of $a$, and denote it by $\operatorname{supp}(a)$, to be the unique smallest subset of $T$ such that $\left\{a_{0}, a_{1}, \ldots, a_{n-1}\right\}$ is $(\mathbb{F} \cup \operatorname{supp}(a))$-definable. Equivalently, $\operatorname{supp}(a)$ is the smallest subset of $T$ such that $\{a\}$ is $(F \cup \operatorname{supp}(a))$-definable.

We next define a countable partition of $\mathbb{R}^{n}$. Actually, we will define a function $\chi$ on $\mathbb{R}^{n}$ having a countable range, and then identify $\chi$ with the countable partition of $\mathbb{R}^{n}$ which it induces. Let $a=\left\langle a_{0}, a_{1}, \ldots, a_{n-1}\right\rangle \in \mathbb{R}^{n}$, and let $\operatorname{supp}(a)=$ $\left\{t_{0}, t_{1}, \ldots, t_{s-1}\right\}$, where $t_{0}<t_{1}<\cdots<t_{s-1}$. Let $q_{0}, q_{1}, \ldots, q_{s-1}, r_{0}, r_{1}, \ldots, r_{s-1} \in$ $\mathbb{Q}$ be such that

$$
q_{0}<t_{0}<r_{0}<q_{1}<t_{1}<r_{1}<\cdots<q_{s-1}<t_{s-1}<r_{s-1}
$$

and (as in [16]) such that there is an $\mathbb{F}$-definable, analytic function $f:\left(q_{0}, r_{0}\right) \times$ $\left(q_{1}, r_{1}\right) \times \cdots \times\left(q_{s-1}, r_{s-1}\right) \rightarrow \mathbb{R}^{n}$ which is one-one in each coordinate and is such that $f\left(t_{0}, t_{1}, \ldots, t_{s-1}\right)=a$.

We briefly indicate how such a function $f$ is obtained. For each $i<n$, let $p_{i}\left(y_{0}, y_{1}, \ldots, y_{s-1}, x\right) \in \mathbb{F}\left[y_{0}, y_{1}, \ldots, y_{s-1}, x\right]$ be a polynomial such that $a_{i}$ is a simple root of the polynomial $p_{i}\left(t_{0}, t_{1}, \ldots, t_{s-1}, x\right)$ in $\mathbb{F}\left(t_{0}, t_{1}, \ldots, t_{s-1}\right)[x]$. By the Implicit Function Theorem there is an analytic function $f_{i}: U_{i} \rightarrow V_{i}$ for some open neighborhoods $U_{i}$ of $\left\langle t_{0}, t_{1}, \ldots, t_{s-1}\right\rangle$ and $V_{i}$ of $a_{i}$ such that if $\left\langle y_{0}, y_{1}, \ldots, y_{s-1}\right\rangle \in U_{i}$ and $x \in V_{i}$, then $f_{i}\left(y_{0}, y_{1}, \ldots, y_{s-1}\right)=x$ iff $p_{i}\left(y_{0}, y_{1}, \ldots, y_{s-1}, x\right)=0$. We arrange that $U_{0}=U_{1}=\cdots=U_{n-1}=\left(q_{0}, r_{0}\right) \times\left(q_{1}, r_{1}\right) \times \cdots \times\left(q_{s-1}, r_{s-1}\right)$ for some appropriate $q_{0}, q_{1}, \ldots, q_{s-1}, r_{0}, r_{1}, \ldots, r_{s-1} \in \mathbb{Q}$ and that each $V_{i}$ is $\mathbb{Q}$-definable. Hence, each $f_{i}$ is $\mathbb{F}$-definable. We can also arrange that $f_{i}$ is one-one in the $j$ th coordinate for those $j<s$ for which $t_{j} \in \operatorname{supp}\left(a_{i}\right)$. Now let $f=\left\langle f_{0}, f_{1}, \ldots, f_{n-1}\right\rangle$. Clearly, $f$ is as required. Let $D$ be the domain of $f$.

To complete the definition of $\chi$, let $\chi(a)=\left\langle f, G\left(\left\{t_{0}, t_{1}, \ldots, t_{s-1}\right\}\right), N\right\rangle$, where $H\left(\left\{t_{0}, t_{1}, \ldots, t_{s-1}\right\}\right)=\left\{t_{j}: j \in N\right\}$. Clearly, $\chi$ has a countable range.

We now consider a $(k, n)$-ary polynomial $p\left(x_{0}, x_{1}, \ldots, x_{k-1}\right) \in \mathbb{F}\left[x_{0}, x_{1}, \ldots, x_{k-1}\right]$ which is not avoided by $\chi$. We will show that $p\left(x_{0}, x_{1}, \ldots, x_{k-1}\right)$ is not $m$-avoidable. Let $a_{0}, a_{1}, \ldots, a_{k-1} \in \mathbb{R}^{n}$ be distinct such that $p\left(a_{0}, a_{1}, \ldots, a_{k-1}\right)=0$ and $\chi\left(a_{0}\right)=$ $\chi\left(a_{1}\right)=\cdots=\chi\left(a_{k-1}\right)=\langle f, d, N\rangle$. Let $N=\left\{j_{0}, j_{1}, \ldots, j_{v-1}\right\}$, where $j_{0}<j_{1}<$ $\cdots<j_{v-1}<s$ and $v=\min (s, m)$. Let $\left(q_{0}, r_{0}\right) \times\left(q_{1}, r_{1}\right) \times \cdots \times\left(q_{s-1}, r_{s-1}\right)$ be the domain of $f$. For each $i<k$, let $\operatorname{supp}\left(a_{i}\right)=\left\{t_{i, 0}, t_{i, 1}, \ldots, t_{i, s-1}\right\}$, where $t_{i, j} \in\left(q_{j}, r_{j}\right)$. (Since $k \geq 2$, it must be that $s \geq 1$.) Therefore, by conditions (1)-(4) of Lemma 2.3 $G\left(\left\{t_{i, 0}, t_{i, 1}, \ldots, t_{i, s-1}\right\}\right)=d$ and $H\left(\left\{t_{i, 0}, t_{i, 1}, \ldots, t_{i, s-1}\right\}\right)=$ $\left\{t_{i, j}: j \in N\right\}$.

It was remarked after Definition 1.1(1) that the open cube $(0,1)^{m}$ that occurs in that definition could be replaced by any other open $m$-box. For this proof, we will use the open $v$-box $B=\left(q_{j_{0}}, r_{j_{0}}\right) \times\left(q_{j_{1}}, r_{j_{1}}\right) \times \cdots \times\left(q_{j_{v-1}}, r_{j_{v-1}}\right)$. Thus, we will show that $p\left(x_{0}, x_{1}, \ldots, x_{k-1}\right)$ is not $v$-avoidable; but then Proposition 1.2 will imply that it is not $m$-avoidable.

Let $g: B \rightarrow \mathbb{R}^{n}$ be defined by $g\left(x_{0}, x_{1}, \ldots, x_{v-1}\right)=f\left(y_{0}, y_{1}, \ldots, y_{s-1}\right)$, where

$$
y_{j}= \begin{cases}x_{i} & \text { if } j=j_{i}, \\ t_{0 j} & \text { if } j \notin N .\end{cases}
$$


For each $i<k$, let $e_{i}=\left\langle t_{i j_{0}}, t_{i j_{1}}, \ldots, t_{i j_{v-1}}\right\rangle \in B$. By Lemma [2.3, the $e_{i}$ 's are distinct. We need to show that for each coordinately induced $\alpha: B \rightarrow B$, $p\left(g \alpha\left(e_{0}\right), g \alpha\left(e_{1}\right), \ldots, g \alpha\left(e_{k-1}\right)\right)=0$.

Consider an arbitrary coordinately induced $\alpha: B \rightarrow B$, where $\alpha\left(x_{0}, x_{1}, \ldots, x_{v-1}\right)$ $=\left\langle\alpha_{0}\left(x_{0}\right), \alpha_{1}\left(x_{1}\right), \ldots, \alpha_{v-1}\left(x_{v-1}\right)\right\rangle$. Let $\beta: D \rightarrow D$ be the coordinately induced function where $\beta\left(y_{0}, y_{1}, \ldots, y_{s-1}\right)=\left\langle\beta_{0}\left(y_{0}\right), \beta_{1}\left(y_{1}\right), \ldots, \beta_{s-1}\left(y_{s-1}\right)\right\rangle$ and

$$
\beta_{j}\left(y_{j}\right)= \begin{cases}\alpha_{i}\left(y_{j}\right) & \text { if } j=j_{i}, \\ t_{0 j} & \text { if } j \notin N .\end{cases}
$$

Consider the $\mathbb{F}$-definable analytic function $h: D^{k} \rightarrow \mathbb{R}$, where

$$
\begin{aligned}
& h\left(y_{0,0}, y_{0,1}, \ldots, y_{0, s-1}, \ldots, y_{k-1,0}, y_{k-1,1}, \ldots, y_{k-1, s-1}\right) \\
& \quad=p\left(f\left(y_{0,0}, y_{0,1}, \ldots, y_{0, s-1}\right), \ldots, f\left(y_{k-1,0}, y_{k-1,1}, \ldots, y_{k-1, s-1}\right)\right) .
\end{aligned}
$$

Then $h\left(t_{0,0}, t_{0,1}, \ldots, t_{0, s-1}, \ldots, t_{k-1,0}, t_{k-1,1}, \ldots, t_{k-1, s-1}\right)=p\left(a_{0}, a_{1}, \ldots, a_{s-1}\right)=$ 0. Then Lemma 2.4 implies that

$$
p\left(f \beta\left(t_{0,0}, t_{0,1}, \ldots, t_{0, s-1}\right), \ldots, f \beta\left(t_{k-1,0}, t_{k-1,1}, \ldots, t_{k-1, s-1}\right)\right)=0 .
$$

To complete this proof, we see that

$$
\begin{aligned}
p(g \alpha & \left.\left(e_{0}\right), g \alpha\left(e_{1}\right), \ldots, g \alpha\left(e_{k-1}\right)\right) \\
& =p\left(g\left(\alpha_{0}\left(t_{0, j_{0}}\right), \ldots, \alpha_{v-1}\left(t_{0, j_{v-1}}\right)\right), \ldots, g\left(\alpha_{0}\left(t_{k-1, j_{0}}\right), \ldots, \alpha_{v-1}\left(t_{k-1, j_{v-1}}\right)\right)\right) \\
& =p\left(g\left(\beta_{j_{0}}\left(t_{0, j_{0}}\right), \ldots, \beta_{j_{v-1}}\left(t_{0, j_{v-1}}\right)\right), \ldots, g\left(\beta_{j_{0}}\left(t_{k-1, j_{0}}\right), \ldots, \beta_{j_{v-1}}\left(t_{k-1, j_{v-1}}\right)\right)\right) \\
& =p\left(f\left(\beta_{0}\left(t_{0,0}\right), \ldots, \beta_{s-1}\left(t_{0, s-1}\right)\right), \ldots, f\left(\beta_{0}\left(t_{k-1,0}\right), \ldots, \beta_{s-1}\left(t_{k-1, s-1}\right)\right)\right) \\
& =p\left(f \beta\left(t_{0,0}, \ldots, t_{0, s-1}\right), \ldots, f \beta\left(t_{k-1,0}, \ldots, t_{k-1, s-1}\right)\right)=0 .
\end{aligned}
$$

Proof of Theorem 0.5 . This is just like the proof of Theorem 0.4 , only easier because we do not need Lemma 2.3. Proceed as in the proof of Theorem 0.4 , but now define the partition so that $\chi(a)=f$.

\section{A Conjecture}

A consequence of a proof of the conjecture presented in this section would be an improvement to Theorem 0.2 We would then have that the set of avoidable polynomials over $\mathbb{Q}$ is recursive.

Definition 3.1. Let $\kappa$ be a cardinal number (either finite or infinite) and let $k, n, m<\omega$. Then the $(k, n)$-ary polynomial $p\left(x_{0}, x_{1}, \ldots, x_{k-1}\right)$ over $\mathbb{R}$ is $(\kappa, m)$ irreflexive if, whenever $|X| \geq \kappa, g: X^{m} \rightarrow \mathbb{R}^{n}$ is one-one in each coordinate and $e_{0}, e_{1}, \ldots, e_{k-1} \in \omega^{m}$ are distinct, then there is a coordinately induced $\alpha: \omega^{m} \rightarrow$ $X^{m}$ such that $p\left(g \alpha\left(e_{0}\right), g \alpha\left(e_{1}\right), \ldots, g \alpha\left(e_{k-1}\right)\right) \neq 0$.

Some simple consequences of this definition are contained in the next proposition.

Proposition 3.2. (1) If $\lambda<\kappa$, then each $(\lambda, m)$-irreflexive polynomial is $(\kappa, m)$ irreflexive.

(2) A polynomial is irreflexive iff it is $(1, m)$-irreflexive iff it is $(1,1)$-irreflexive.

(3) If $r<m$, then each $(\kappa, m)$-irreflexive polynomial is $(\kappa, r)$-irreflexive.

(4) Every polynomial is $\left(\left(2^{\aleph_{0}}\right)^{+}, m\right)$-irreflexive.

(5) No polynomial is $(0, m)$-irreflexive.

(6) Every $\left(2^{\aleph_{0}}, m\right)$-irreflexive polynomial is m-avoidable.

The conjecture is stronger than the converse of Proposition [3.2 6 ). 
Conjecture 3.3. For each $(k, n)$-ary polynomial $p\left(x_{0}, x_{1}, \ldots, x_{k-1}\right)$ there exists $N<\omega$ such that if $p\left(x_{0}, x_{1}, \ldots, x_{k-1}\right)$ is $m$-avoidable, then it is $(N, m)$-irreflexive.

It would follow from Conjecture [3.3, by the decidability of $\operatorname{Th}(\mathbb{R},+, \cdot, \leq, 0,1)$, that the set of $m$-avoidable polynomials is recursively enumerable (uniformly in $m$ ). Thus, irrespective of the cardinality of the continuum, it would follow from Corollaries 0.6 and 0.7 and Proposition 1.3 that the set of avoidable polynomials over $\mathbb{Q}$ is recursive. It would also follow that the requirement in Definition 1.1 1 ) that $g$ be definable could be dropped.

\section{REFERENCES}

[1] R. Benedetti and J.-J. Risler, Real Algebraic and Semi-algebraic Sets, Hermann, Paris, 1990. MR 91j:14045

[2] J. Ceder, Finite subsets and countable decompositions of Euclidean spaces, Rev. Roumaine Math. Pures Appl. 14 (1969), 1247-1251. MR 41:1958

[3] R. O. Davies, Partitioning the plane into denumerably many sets without repeated distances, Proc. Cambridge Philos. Soc. 72 (1972), 179-183. MR 45:3662

[4] P. Erdős and A. Hajnal, On chromatic number of graphs and set systems, Acta Math. Hungar. 17 (1966), 61-99. MR 41:8294

[5] P. Erdős, A. Hajnal, A. Máté and R. Rado, Combinatorial Set Theory; Partition Relation for Cardinals, North-Holland, Amsterdam, 1984. MR 87g:04002

[6] P. Erdős and S. Kakutani, On non-denumerable graphs, Bull. Amer. Math. Soc. 49 (1943), 457-461. MR 4:249f

[7] P. Erdős and P. Komjáth, Countable decompositions of $\mathbb{R}^{2}$ and $\mathbb{R}^{3}$, Discrete \& Comput. Geom. 5 (1990), 325-331. MR 91b:04002

[8] P. Komjáth, Tetrahedron free decompositions of $\mathbb{R}^{3}$, Bull. London Math. Soc. 23 (1991), 116-120. MR 92i:52008

[9] P. Komjáth, The master coloring, Comptes Rendus Mathématique de l'Academie des Sciences, la Société Royale du Canada 14 (1992), 181-182. MR 94a:05076

[10] P. Komjáth, A decomposition theorem for $\mathbb{R}^{n}$, Proc. Amer. Math. Soc. 120 (1994), 921-927. MR 94h:04005

[11] P. Komjáth, Partitions of vector spaces, Period. Math. Hungar. 28 (1994), 187-193. MR 95k:04004

[12] P. Komjáth, personal letter to the author, 1992.

[13] K. Kunen, Partitioning Euclidean space, Math. Proc. Cambridge Philos. Soc. 102 (1987), 379-383. MR 88i:05014

[14] J. H. Schmerl, Partitioning Euclidean space, Discrete \& Comp. Geom. 10 (1993), 101-106. MR 94e:05262

[15] J. H. Schmerl, Triangle-free partitions of Euclidean space, Bull. London Math. Soc. 26 (1994), 483-486. MR 95i:52019

[16] J. H. Schmerl, Countable partitions of Euclidean space, Math. Proc. Cambridge Philos. Soc. 120 (1996), 7-12. MR 96k:52011

[17] L. van den Dries, Alfred Tarski's elimination theory for real closed fields, J. Symbolic Logic 53 (1988), 7-19. MR 89h:01040

Department of Mathematics, University of Connecticut, Storrs, Connecticut 062693009

E-mail address: schmerl@math.uconn.edu 